\title{
BMC Infectious Diseases reviewer acknowledgement 2015
}

Hilary Logan

\section{Contributing reviewers}

The editors of BMC Infectious Diseases would like to thank all our reviewers who have contributed to the journal in Volume 15 (2015).

Fernando Abad-Franch

Stephen Abedon

USA

Florence Abravanel

France

Muhammad Abubakar

Pakistan

Sazaly Abubakar

Malaysia

Chad Achenbach

USA

Olivia Achonduh-Atijegbe

Cameroon

Ishag Adam

Sudan

Ayola Akim Adegnika

Germany

Javier Afeltra

Argentina

Farhat Afrin

Saudi Arabia

Rupesh Agrawal

Singapore

Golo Ahlenstiel

Australia
Hyo Yeong Ahn

Korea, South

Aima Ahonkhai

USA

Mustapha Ahsaini

Morocco

Vineet Ahuja

India

Maria Aigner

Austria

Marta Aires-De-Sousa

Portugal

Katherine Ajdukiewicz

UK

Sitara Ajjampur

India

MJ Alam

USA

Isabelle Albert

France

Werner Albrich

Switzerland

David Alexander

Canada

Vangelis Alexiou

Greece
Nahid Ali

India

Bachti Alisjahbana

Indonesia

Nicolaos Almyroudis

USA

Carolyn Alonso

USA

Thomas Althaus

Thailand

Benjamin Althouse

USA

Cosme Alvarado-Esquivel

Mexico

Francisco Álvarez García

Spain

Carlos Roberto Alves

Brazil

Gayatri Amirthalingam

UK

Rune Andersson

Sweden

Warren Andiman

USA

Anuska Andjelkovic Zochowska USA

Correspondence: hilary.logan@biomedcentral.com

BioMed Central, Floor 6, 236 Gray's Inn Road, London WC1X 8HB, UK 


\begin{tabular}{|c|c|c|}
\hline $\begin{array}{l}\text { Anton Andonov } \\
\text { Canada }\end{array}$ & $\begin{array}{l}\text { Elizabeth Ashley } \\
\text { Thailand }\end{array}$ & $\begin{array}{l}\text { David Banach } \\
\text { USA }\end{array}$ \\
\hline $\begin{array}{l}\text { Genevieve Andre-Fontaine } \\
\text { France }\end{array}$ & $\begin{array}{l}\text { Lelisa Assebe } \\
\text { Ethiopia }\end{array}$ & $\begin{array}{l}\text { Niaz Banaei } \\
\text { USA }\end{array}$ \\
\hline $\begin{array}{l}\text { Nick Andrews } \\
\text { UK }\end{array}$ & $\begin{array}{l}\text { Ross Atkinson } \\
\text { UK }\end{array}$ & $\begin{array}{l}\text { Ritu Banerjee } \\
\text { USA }\end{array}$ \\
\hline $\begin{array}{l}\text { Wim Ang } \\
\text { The Netherlands }\end{array}$ & $\begin{array}{l}\text { Enas Attia } \\
\text { Egypt }\end{array}$ & $\begin{array}{l}\text { Pankaj Baral } \\
\text { USA }\end{array}$ \\
\hline $\begin{array}{l}\text { Martin Angelin } \\
\text { Sweden }\end{array}$ & $\begin{array}{l}\text { Christian Auer } \\
\text { Switzerland }\end{array}$ & $\begin{array}{l}\text { Tristan Barber } \\
\text { UK }\end{array}$ \\
\hline $\begin{array}{l}\text { Mehar Angez } \\
\text { Pakistan }\end{array}$ & $\begin{array}{l}\text { Peter Axelrod } \\
\text { USA }\end{array}$ & $\begin{array}{l}\text { Dulce Barbosa } \\
\text { Brazil }\end{array}$ \\
\hline $\begin{array}{l}\text { Francois Angoulvant } \\
\text { France }\end{array}$ & $\begin{array}{l}\text { Chaw Aye } \\
\text { Australia }\end{array}$ & $\begin{array}{l}\text { Francis Barin } \\
\text { France }\end{array}$ \\
\hline $\begin{array}{l}\text { Brian Angus } \\
\text { UK }\end{array}$ & $\begin{array}{l}\text { Andrew Azman } \\
\text { USA }\end{array}$ & $\begin{array}{l}\text { Timothy Barkham } \\
\text { Singapore }\end{array}$ \\
\hline $\begin{array}{l}\text { Filippo Ansaldi } \\
\text { Italy }\end{array}$ & $\begin{array}{l}\text { Lilian Azzopardi } \\
\text { Malta }\end{array}$ & $\begin{array}{l}\text { Gavin Barlow } \\
\text { UK }\end{array}$ \\
\hline $\begin{array}{l}\text { Constantine Antonopoulos } \\
\text { Greece }\end{array}$ & $\begin{array}{l}\text { Subash Babu } \\
\text { India }\end{array}$ & $\begin{array}{l}\text { Ruanne Barnabas } \\
\text { USA }\end{array}$ \\
\hline $\begin{array}{l}\text { Victor H Aquino } \\
\text { Brazil }\end{array}$ & $\begin{array}{l}\text { John Baddley } \\
\text { USA }\end{array}$ & $\begin{array}{l}\text { Arvind Baronia } \\
\text { India }\end{array}$ \\
\hline $\begin{array}{l}\text { Amber Arain } \\
\text { Belgium }\end{array}$ & $\begin{array}{l}\text { Wolfgang Baeumer } \\
\text { USA }\end{array}$ & $\begin{array}{l}\text { Naor Bar-Zeev } \\
\text { Malawi }\end{array}$ \\
\hline $\begin{array}{l}\text { Minerva Arce-Fonseca } \\
\text { Mexico }\end{array}$ & $\begin{array}{l}\text { Nathan Bahr } \\
\text { USA }\end{array}$ & $\begin{array}{l}\text { Matteo Bassetti } \\
\text { UK }\end{array}$ \\
\hline $\begin{array}{l}\text { Carmen Ardanuy } \\
\text { Spain }\end{array}$ & $\begin{array}{l}\text { Zhenggang Bai } \\
\text { China }\end{array}$ & $\begin{array}{l}\text { Monica Basso } \\
\text { Italy }\end{array}$ \\
\hline $\begin{array}{l}\text { Katherine Arden } \\
\text { Australia }\end{array}$ & $\begin{array}{l}\text { Anda Baicus } \\
\text { Romania }\end{array}$ & $\begin{array}{l}\text { Francisco Bastos } \\
\text { Brazil }\end{array}$ \\
\hline $\begin{array}{l}\text { Alicia Arechavala } \\
\text { Argentina }\end{array}$ & $\begin{array}{l}\text { J Baillie } \\
\text { UK }\end{array}$ & $\begin{array}{l}\text { Matthew Bates } \\
\text { UK }\end{array}$ \\
\hline $\begin{array}{l}\text { Jorge Arevalo } \\
\text { Brazil }\end{array}$ & $\begin{array}{l}\text { Francis Bajunirwe } \\
\text { Uganda }\end{array}$ & $\begin{array}{l}\text { Wagner Luiz Batista } \\
\text { Brazil }\end{array}$ \\
\hline $\begin{array}{l}\text { Emmanuel Arinaitwe } \\
\text { Uganda }\end{array}$ & $\begin{array}{l}\text { Brook Baker } \\
\text { USA }\end{array}$ & $\begin{array}{l}\text { Deepak Batura } \\
\text { UK }\end{array}$ \\
\hline $\begin{array}{l}\text { Frederick Armah } \\
\text { UK }\end{array}$ & $\begin{array}{l}\text { Mirjam Irene Bakker } \\
\text { The Netherlands }\end{array}$ & $\begin{array}{l}\text { Abebe Genetu Bayih } \\
\text { Canada }\end{array}$ \\
\hline $\begin{array}{l}\text { George Armah } \\
\text { Ghana }\end{array}$ & $\begin{array}{l}\text { Manju Bala } \\
\text { India }\end{array}$ & $\begin{array}{l}\text { Pontius Bayo } \\
\text { Uganda }\end{array}$ \\
\hline $\begin{array}{l}\text { Janet Arno } \\
\text { USA }\end{array}$ & $\begin{array}{l}\text { Ludmila Baltazar } \\
\text { USA }\end{array}$ & $\begin{array}{l}\text { Ken Beagley } \\
\text { Australia }\end{array}$ \\
\hline $\begin{array}{l}\text { Peter H Asdahl } \\
\text { Denmark }\end{array}$ & $\begin{array}{l}\text { Alasdair Bamford } \\
\text { UK }\end{array}$ & $\begin{array}{l}\text { Karsten Becker } \\
\text { Germany }\end{array}$ \\
\hline
\end{tabular}


Soeren Becker

Germany

Richard Bedell

USA

Rodolfo Begue

USA

Pedro Belda-Ferre

Spain

Charlotte Bell

Australia

Steve Bellan

USA

Silvana Belo

Portugal

Alexia Belperron

USA

Andrea Benedetti

Canada

Thomas Bénet

France

Abdelouaheb Bennani

Morocco

Kim Benschop

The Netherlands

Guillaume Béraud

France

Bradford Berges

USA

Dennis Bergmans

The Netherlands

Louis Bernard

France

Jose Ignacio Bernardino

Spain

Mariolga Berrizbeitia

Venezuela

Khalid Beshir

UK

Debra Bessen

USA

Andrew David Beswick

UK

Sarah Bevins

USA
Natalie Beylis

South Africa

Matthew Beymer

USA

N Bhadelia

USA

Sanjay Bhagani

UK

Preeti Bharaj

India

Sandeep Bharaswadikar

India

Amrita Bharat

Canada

Claver Bhunu

Zimbabwe

Sibhatu Biadgilign

Ethiopia

Lena Biehl

Germany

Marius Birlea

USA

Hashem Bishara

Israel

Debasis Biswas

India

Jason Blackard

USA

José-Ramón Blanco

Spain

Meridith Blevins

USA

Lucie Blok

The Netherlands

Frank Bloos

Germany

Stijn Blot

Belgium

Thomas Bock

Germany

Barbara Body

USA

Debrah Boeras

USA
Almut Böer-Auer

Germany

Vicente Boix

Spain

Jesper Bonde

Denmark

Maryline Bonnet

Switzerland

Meatherall Bonnie

Canada

Rémy Bonnin

France

Celestino Bonura

Italy

Andre Boonstra

The Netherlands

Timothy Booth

Canada

Paola Borella

Italy

Vanni Borghi

Italy

Philipp Bosshard

Switzerland

Julie Bottero

France

Valerie Bouchez

France

Yap Boum

Uganda

Roberto Bovo

Italy

Lyudmila Boyanova

Bulgaria

Anders Boyd

France

Sebastien Boyer

Madagascar

Daniel Boyle

USA

Nicole Boyle

USA

Steven Bradfute

USA 
Silvia Bressan

Italy

Richard Brindle

UK

Claire Bristow

USA

Murilo Britto

Brazil

Etienne Brochot

France

Claudia Brodskyn

Brazil

Ruth Broering

Germany

David Broniatowski

USA

Barbara Brown-Elliot

USA

Roberta Bruhn

USA

Sylvia Bruisten

The Netherlands

Fabrice Bruneel

France

Vincent Bruno

USA

Cheryl Brunton

New Zealand

Nele Brusselaers

Sweden

Juliet Bryant

UK

Udo Buchholz

Germany

Maria Jose Buitrago

Spain

Ann Burchell

Canada

Emily Burger

Norway

David Burns

USA

Bettina Buttaro

USA
Steve Bynon

USA

Andrea Maurizio Cabibbe Italy

Omar Caceres

Peru

Rui Cai

The Netherlands

Morena Caira

Italy

Andrea Calcagno

Italy

Michael Calderwood

USA

Giorgio Calisti

UK

Greg Calligaro

South Africa

Angela Campbell

USA

Adrian Canizalez

Mexico

Angela Cannas

Italy

Stefania Cannizzo

Italy

Jorge Cano

UK

Cristina Canteros

Argentina

Edmund Capparelli

USA

Federico Capparelli

Argentina

Luciana Cardoso

Brazil

Philip Carling

USA

Francesca Carozzi

Italy

Fabrice Carrat

France

Patrizia Carrieri

France
Enitan Carrol

UK

Cecilia Godoy Carvalhaes Brazil

Edgar M Carvalho

Brazil

Peg Carver

USA

Jose Casado

Spain

Antonio Cascio

Italy

Susan Cassels

USA

Antonella Castagna

Italy

Sandrine Castelain

France

Barbara Castelnuovo

Uganda

Maria Catania

Italy

Sagnelli Caterina

Italy

Francesco Cattel

Italy

Serena Cavallero

Italy

Peter Cegielski

USA

Cornelia Ceianu

Romania

Robert Centor

USA

Maria Cernada

Spain

Gabriela Certad

France

Guillaume Chabot-Couture USA

Dave Chadee

Trinidad and Tobago

Shua Chai

USA 


\begin{tabular}{|c|c|c|}
\hline $\begin{array}{l}\text { Andrew Chan } \\
\text { Germany }\end{array}$ & $\begin{array}{l}\text { Qijun Chen } \\
\text { China }\end{array}$ & $\begin{array}{l}\text { Chin-Cheng Chou } \\
\text { Taiwan }\end{array}$ \\
\hline $\begin{array}{l}\text { Duncan Chanda } \\
\text { Zambia }\end{array}$ & $\begin{array}{l}\text { Yi-Ming Arthur Chen } \\
\text { Taiwan }\end{array}$ & $\begin{array}{l}\text { Eric Chow } \\
\text { Australia }\end{array}$ \\
\hline $\begin{array}{l}\text { Pranatharthi Chandrasekar } \\
\text { USA }\end{array}$ & $\begin{array}{l}\text { You-Hua Chen } \\
\text { Canada }\end{array}$ & $\begin{array}{l}\text { Abhay Chowdhary } \\
\text { India }\end{array}$ \\
\hline $\begin{array}{l}\text { Lisa Chang } \\
\text { Australia }\end{array}$ & $\begin{array}{l}\text { Zhi-Min Chen } \\
\text { China }\end{array}$ & $\begin{array}{l}\text { Abhijit Chowdhury } \\
\text { India }\end{array}$ \\
\hline $\begin{array}{l}\text { Rudragouda Channappanavar } \\
\text { USA }\end{array}$ & $\begin{array}{l}\text { Pin-Nan Cheng } \\
\text { Taiwan }\end{array}$ & $\begin{array}{l}\text { Christina Christoffersen } \\
\text { Denmark }\end{array}$ \\
\hline $\begin{array}{l}\text { Francois Chappuis } \\
\text { Switzerland }\end{array}$ & $\begin{array}{l}\text { Shih-Lung Cheng } \\
\text { Taiwan }\end{array}$ & $\begin{array}{l}\text { Helen Chu } \\
\text { USA }\end{array}$ \\
\hline $\begin{array}{l}\text { Esmita Charani } \\
\text { UK }\end{array}$ & $\begin{array}{l}\text { Vincent Cheng } \\
\text { Hong Kong }\end{array}$ & $\begin{array}{l}\text { Hin Chu } \\
\text { Hong Kong }\end{array}$ \\
\hline $\begin{array}{l}\text { Patrick Charles } \\
\text { Australia }\end{array}$ & $\begin{array}{l}\text { Fanny Chereau } \\
\text { France }\end{array}$ & $\begin{array}{l}\text { Anthony Chua } \\
\text { Singapore }\end{array}$ \\
\hline $\begin{array}{l}\text { Carmen Charlton } \\
\text { Canada }\end{array}$ & $\begin{array}{l}\text { Stephane Chevaliez } \\
\text { France }\end{array}$ & $\begin{array}{l}\text { Arlene Chua } \\
\text { Switzerland }\end{array}$ \\
\hline $\begin{array}{l}\text { PH Chau } \\
\text { Hong Kong }\end{array}$ & $\begin{array}{l}\text { Claire Chewapreecha } \\
\text { UK }\end{array}$ & $\begin{array}{l}\text { Voo Teck Chuan } \\
\text { USA }\end{array}$ \\
\hline $\begin{array}{l}\text { Ruchir Chavada } \\
\text { Australia }\end{array}$ & $\begin{array}{l}\text { Jung-Yien Chien } \\
\text { Taiwan }\end{array}$ & $\begin{array}{l}\text { Abrar Chughtai } \\
\text { Australia }\end{array}$ \\
\hline $\begin{array}{l}\text { Porntip Chavalitshewinkoon-Petmitr } \\
\text { Thailand }\end{array}$ & $\begin{array}{l}\text { Ng Lee Ching } \\
\text { Singapore }\end{array}$ & $\begin{array}{l}\text { Linda Chui } \\
\text { Canada }\end{array}$ \\
\hline $\begin{array}{l}\text { Kalyan Chavda } \\
\text { USA }\end{array}$ & $\begin{array}{l}\text { Nyasha Chin'Ombe } \\
\text { Zimbabwe }\end{array}$ & $\begin{array}{l}\text { Ju-Young Chung } \\
\text { South Korea }\end{array}$ \\
\hline $\begin{array}{l}\text { Guilherme Chaves } \\
\text { Brazil }\end{array}$ & $\begin{array}{l}\text { EM Chisati } \\
\text { Malawi }\end{array}$ & $\begin{array}{l}\text { Gavin John Churchyard } \\
\text { South Africa }\end{array}$ \\
\hline $\begin{array}{l}\text { Phaikyeong Cheah } \\
\text { Thailand }\end{array}$ & $\begin{array}{l}\text { Mohammod Chisti } \\
\text { Bangladesh }\end{array}$ & $\begin{array}{l}\text { Miquel Viñas Ciordia } \\
\text { Spain }\end{array}$ \\
\hline $\begin{array}{l}\text { Cynthia Chee } \\
\text { Singapore }\end{array}$ & $\begin{array}{l}\text { Yen-Cheng Chiu } \\
\text { Taiwan }\end{array}$ & $\begin{array}{l}\text { Ivana Cirkovic } \\
\text { Serbia }\end{array}$ \\
\hline $\begin{array}{l}\text { Chih-Jung Chen } \\
\text { Taiwan }\end{array}$ & $\begin{array}{l}\text { Nikoloz Chkhartishvili } \\
\text { Georgia }\end{array}$ & $\begin{array}{l}\text { Mareli Claassens } \\
\text { South Africa }\end{array}$ \\
\hline $\begin{array}{l}\text { Haiying Chen } \\
\text { China }\end{array}$ & $\begin{array}{l}\text { Maciej Piotr Chlebicki } \\
\text { Singapore }\end{array}$ & $\begin{array}{l}\text { Jan Clement } \\
\text { Belgium }\end{array}$ \\
\hline $\begin{array}{l}\text { Liang Chen } \\
\text { USA }\end{array}$ & $\begin{array}{l}\text { Jun Yong Choi } \\
\text { South Korea }\end{array}$ & $\begin{array}{l}\text { Gary Clifford } \\
\text { France }\end{array}$ \\
\hline $\begin{array}{l}\text { Lizhang Chen } \\
\text { China }\end{array}$ & $\begin{array}{l}\text { Sidharth Chopra } \\
\text { India }\end{array}$ & $\begin{array}{l}\text { Nathan Clumeck } \\
\text { Belgium }\end{array}$ \\
\hline $\begin{array}{l}\text { Marcus Chen } \\
\text { Australia }\end{array}$ & $\begin{array}{l}\text { Teena Chopra } \\
\text { USA }\end{array}$ & $\begin{array}{l}\text { Patrick Coffie } \\
\text { Côte D'Ivoire }\end{array}$ \\
\hline $\begin{array}{l}\text { Po-Yen Chen } \\
\text { Taiwan }\end{array}$ & $\begin{array}{l}\text { Vineet Chopra } \\
\text { USA }\end{array}$ & $\begin{array}{l}\text { Adam Cohen } \\
\text { South Africa }\end{array}$ \\
\hline
\end{tabular}


Bevin Cohen

USA

Cheryl Cohen

South Africa

Donn Colby

Viet Nam

Brenda Coleman

Canada

Luis Collado González

Chile

Julio Collazos

Spain

Paul Collini

UK

Arnaldo Colombo

Brazil

Philippe Colson

France

Martie Conradie

South Africa

Carlo Contini

Italy

James Conway

USA

Alex Cook

Singapore

Paul Cook

USA

Fiona Cooke

UK

Barry Cookson

USA

Yacoob Coovadia

South Africa

Nicola Coppola

Italy

Marli Cordeiro

Brazil

Rossana Cordeiro

Brazil

Victor Max Corman

Germany

Alyssa Cornall

Australia
Markus Cornberg

Germany

Carlos Costa

Brazil

Claudio Costantino

Italy

Jean Tenena Coulibaly

Cote D'Ivoire

Sonali Coulter

Australia

Kevin Couper

UK

Benjamin Cowling

Hong Kong

Peter Coyle

UK

Emanuele Cozzani

Italy

Alessandro Cozzi-Lepri

UK

Duncan Cranendonk

The Netherlands

Olaf L Cremer

The Netherlands

Amelieke Cremers

The Netherlands

Anne-Claude Crémieux

France

Jacob Creswell

Switzerland

Pablo Cruces

Chile

Nancy Crum-Cianflone

USA

Isra Cruz

Switzerland

Robert Cunney

Ireland

Jessica Cusato

Italy

Brian Custer

USA

Jeffery Cutter

Singapore
Nayle Da Silva

Brazil

Prabin Dahal

UK

Gunnar Dahlen

Sweden

Fredrick Dahlgren

USA

Chia-Yen Dai

Taiwan

Tine Dalby

Denmark

Fortunato D'Ancona Italy

Nick Daneman

Canada

Zhisheng Dang

Japan

Ina Danquah

Germany

Dilip Kumar Das

New Zealand

Pranab Das

UK

Michael David

USA

Christopher Davies

USA

D Davis

USA

Mohamed Ali Daw

Libya

Rod Dawson

South Africa

Wu De

China

Bertille de Barbeyrac

France

Birgitte de Blasio

Norway

Thomas de Broucker

France

Gabriella De Carli

Italy 
Elena De Carolis

Italy

Daan de Gouw

USA

Jokin de Irala

Spain

Gennaro De Pascale

Italy

Francesco Giuseppe de Rosa

Italy

Silvia De Sanjose

Spain

Henry de Vries

The Netherlands

Jan De Waele

Belgium

Chiara De Waure

Italy

G Ardine de Wit

The Netherlands

Fantahun Defeneh

Ethiopia

Abraham Degarege

Ethiopia

Takashi Deguchi

Japan

Valerio Del Bono

Italy

Annarosa Del Mistro

Germany

Gilda Maria Barbaro Del Negro

Brazil

Claudia Del Vecchio

Italy

Cynthia Delgado

USA

Casper Den Heijer

The Netherlands

Licia Denti

Italy

Moutaz Derbala

Qatar

Amare Deribew

Kenya
T Derrick

UK

Benoît Dervaux

France

Jean-Claude Desenclos

France

Sophie Desmonde

France

Roger Detels

USA

Gabriella D'Ettorre

Italy

Gregor Devine

Australia

Ashraf Dewan

Australia

Sorabh Dhar

USA

Daniel Dhumeaux

France

Stefano Di Bella

Italy

Antonio Di Biagio

Italy

Alvaro Diaz

Uruguay

Roland Diel

Germany

Matthew Dietz

USA

Javier Diez-Domingo

Spain

Maria Cecilia Dignani

Argentina

Lenie Dijkshoorn

The Netherlands

Jo-Anne Dillon

Canada

Andrew Dinardo

USA

Jodie Dionne-Odom

USA

Katharina Diouf

Germany
Olgica Djurkovic-Djakovic

Serbia

Russell Dlae

Australia

Hans-Wilhelm Doerr

Germany

Thomas Doker

USA

Changzheng Dong

China

Thomas Dorlo

The Netherlands

Marcus Dorner

UK

Horng-Yunn Dou

Taiwan

Paul Drain

USA

Alison Drake

USA

Michèle Dramaix-Wilmet

Belgium

Michel Drancourt

France

Johannes Dreesman

Germany

Robert Dreibelbis

USA

Dimitri Drekonja

USA

Steven Drews

Canada

Nicholas Drey

UK

Maria D'Souza

India

Mignon Du Plessis

South Africa

Nancy Duah

Ghana

Raquel Duarte

Portugal

Alka Dubey

India 
Jean-Charles Duclos-Vallée

France

Birgitta Duim

The Netherlands

Anne Dulioust

France

Eileen Dunne

Australia

Herve Dupont

France

Agnès Dupret-Bories

France

Emanuele Durante Mangoni

Italy

Solange Durao

South Africa

Kerina Duri

Zimbabwe

Michael Durkin

USA

Malcolm Duthie

USA

Richard Dwyer

Sweden

Shetty Ravi Dyavar

USA

Tim Eckmanns

Germany

Obaghe Edeghere

UK

Stefan Edlund

USA

Kathryn Edwards

USA

Simon Edwards

UK

Philippe Eggimann

Switzerland

Peter Eichacker

USA

Lloyd Einsiedel

Australia

Koumavi Didier Ekouevi

Cote D'Ivoire
Kamal El Bissati

USA

Maissa El Raziky

Egypt

Johannes Elias

Germany

Briony Elliott

Australia

George Eluwa

Nigeria

Mohammad Emaneinie Iran

Janice Endsley

USA

Hock-Liew Eng

Taiwan

Mark Enright

UK

Olivier Epaulard

France

Serpil Erdogan

Turkey

Kristine Erlandson

USA

Ananias Escalante

USA

Janne Estill

Switzerland

Concepcion Estivariz

USA

Vicente Estrada

Spain

Charlesnika Evans

USA

Echezona Ezeanolue USA

Massimiliano Fabbiani Italy

Cornelius Faber

Germany

Diego Rodrigues Falci

Brazil

Vicenç Falcó

Spain
Marco Falcone

Italy

Joseph Falkinham

USA

Oana Falup-Pecurariu

Romania

Chi-Tai Fang

Taiwan

Maryam Farooqui

Malaysia

Lanfranco Fattorini

Italy

Saul Faust

UK

Jonathan Feelemyer USA

Thomas Fekete USA

Arnaud Fekkar

France

Jordan Feld

Canada

Jia-Yih Feng

USA

Kevin Fennelly

USA

Peter Ferenci

Austria

Rashida Ferrand

UK

Eliane Ferreira

Brazil

Pedro Ferreira

Japan

James Fielding

Australia

Suzanne Filteau

UK

Katja Fink

Singapore

Ramona Finnie USA

Rebuma Firdessa

USA 
Peter Fischer

USA

Dale Fisher

Singapore

Deirdre Fitzgerald-Hughes

Ireland

Fidelma Fitzpatrick

Ireland

Carolin Fleischmann

Germany

Stephanie Fletcher

Australia

William Flight

UK

Carole Fogg

UK

Brian Foley

USA

Jason Folster

USA

Alex Fonseca

USA

Patrice Forget

Belgium

Giulio Formoso

Italy

Kevin Forsyth

Australia

Gregory Fox

Australia

Henry S Fraimow

USA

Cristiana Franchi

Italy

Eduardo Franco

Canada

Elisabetta Franco

Italy

Simon François

France

Daniel Freedberg

USA

Matthew Freeman

USA
Lucia Freitas

Brazil

Hagen Frickmann

Germany

Jan Friedrich

Canada

Nina Friis-Møller

Denmark

Niels Frimodt-Moller

Denmark

Holly Frost

USA

Chuanxi Fu

China

I Fuentes

Spain

Keiichi Fujiwara

Japan

Francesco Maria Fusco

Italy

Giovanni Gabutti

Italy

Jacques Gaillat

France

Katya Galactionova

Switzerland

Shawn Gale

USA

Jason Gallagher

USA

Chandika Gamage

India

Manoj Gambhir

Australia

Maria Ganczak

Poland

Rita Gander

USA

Sumanth Gandra

India

Qian Gao

China

Maria Garces-Sanchez

Spain
Federico Garcia

Spain

George Garcia

USA

Joao Luis Garcia

Brazil

Alberto García-Basteiro

Mozambique

Guillermo Garcia-Effron

Argentina

Aurora Garcia-Fernandez

Italy

Carlo Garzelli

Italy

Marie Gauthier

France

Philippe Gautret

France

Jenn Geddes

USA

Larrouy Gerald

UK

Dionne Gesink

Canada

Antoine Gessain

France

Laurent Getaz

Switzerland

Corine Geurts Van Kessel

The Netherlands

Fatemeh Ghaffarifar

Iran

Sarah Gheuens

USA

Valeria Ghisetti

Italy

Evangelos Giamarellos-Bourboulis

Greece

Helen Giamarellou

Greece

Cristina Giambi

Italy

Ricardo Gianecini

Argentina 


\begin{tabular}{|c|c|c|}
\hline $\begin{array}{l}\text { Antonino Giarratano } \\
\text { Italy }\end{array}$ & $\begin{array}{l}\text { Daniel Golparian } \\
\text { Sweden }\end{array}$ & $\begin{array}{l}\text { Magdalena Grce } \\
\text { Croatia }\end{array}$ \\
\hline Sebastien Gibot & Andre Gomes & Jason Grebely \\
\hline France & Brazil & Australia \\
\hline Jose Pedro Gil & Jose Gomez-Rial & Giampaolo Greco \\
\hline Sweden & Spain & USA \\
\hline Brad Gilbertson & Cecilia Gonzales-Marin & David Greenberg \\
\hline Australia & UK & USA \\
\hline Mary Ellen Gilder & Julià González Martin & Andrew Greenhill \\
\hline USA & Spain & Australia \\
\hline Francisco Gimenez-Sanchez & Ted Gooley & David Griffith \\
\hline Spain & USA & USA \\
\hline Mario Giobbia & Satish Gopal & Paul Griffiths \\
\hline Italy & Malawi & UK \\
\hline Paolo Giorgi Rossi & Shubha Gopal & Ulla Griffiths \\
\hline Italy & India & UK \\
\hline Delphine Girard & Gregor Gorkiewicz & Andreas H. Groll \\
\hline Switzerland & Austria & Germany \\
\hline Enrico Girardi & Tomasz Gosiewski & Michelle Jennifer Groome \\
\hline Italy & Poland & South Africa \\
\hline Delphine Girlich & Neela Goswami & Charles Grose \\
\hline France & USA & USA \\
\hline Corrado Girmenia & Michihiko Goto & Robert Gross \\
\hline Italy & USA & USA \\
\hline Sophie Githinji & Hannelore Gotz & Gunnar Hischebeth \\
\hline Kenya & The Netherlands & Germany \\
\hline Gustavo Giusiano & Nelesh Govender & Duan Guangcai \\
\hline Argentina & South Africa & China \\
\hline Kathryn Glass & Charitha Gowda & Jeremie Guedj \\
\hline Australia & USA & France \\
\hline Erik Glocker & Maira Goytia & Francois Guerin \\
\hline Germany & USA & France \\
\hline Cyrille Goarant & Dennis Grab & Yan Guex-Crosier \\
\hline New Caledonia & USA & Switzerland \\
\hline Nele Goeyvaerts & Christopher Graber & Thaís Guimarães \\
\hline Belgium & USA & Brazil \\
\hline Alejandra Goldman & Luigi Gradoni & Rajni Gunnala \\
\hline Argentina & Italy & USA \\
\hline Lloyd Goldsamt & Morag Graham & Anuj Gupta \\
\hline USA & Canada & India \\
\hline Edward Goldstein & Lucia Grandiere-Perez & Ravindra Kumar Gupta \\
\hline USA & France & UK \\
\hline Justyna Gołębiewska & James Gray & Rishein Gupta \\
\hline Poland & UK & USA \\
\hline
\end{tabular}


Shashank Gupta

USA

Ankur Gupta-Wright

UK

Juan Pablo Gutierrez

Mexico

Michael Haber

USA

Nagy A. Habib

UK

Emilia Hadziyannis

Greece

Ferry Hagen

The Netherlands

Asrat Hailu

Ethiopia

Behzad Hajarizadeh

Australia

Mahmoud Halablab

Lebanon

Valerie Haley

USA

Ronald Hall

USA

Sebastian Haller

Germany

Gabriel Hamer

USA

Richard Hamilton

USA

Theodore Hammett

USA

Klaus Hamprecht

Germany

Camille Hamula

USA

Yasmeen Hanifa

UK

David Hanna

USA

Margaret Hannan

Ireland

Ann-Brit Eg Hansen

Denmark
Abdul Haque

Pakistan

Syed Haque

India

Ubydul Haque

USA

Pia Hardelid

UK

David Harley

Australia

Guy Harling

USA

P Richard Harrigan

Canada

John Harris

UK

Hans Hartling

USA

Rumina Hasan

Pakistan

Zahra Hasan

Pakistan

Rodrigo Hasbun

USA

Kenji Hashimoto

Japan

Angelos Hatzakis

Greece

Stephen Hawes

USA

Claudia Hawkins

USA

Alastair Hay

UK

Miao He

China

Yu He

USA

Pasco Hearn

Cambodia

Janneke Heijne

The Netherlands

Ghada Helaly

Egypt
Gideon Kofi Helegbe

Ghana

Markus Hell

Austria

Niels Bohse Hendriksen

Denmark

Niel Hens

Belgium

Melissa Herbst-Kralovetz

USA

Sabine Hermans

Uganda

Alexandra Hernandez

USA

Björn Herrmann

Sweden

Jean-Louis Herrmann

France

Cord Heuer

New Zealand

Kirsty Hewitt

UK

Scott Heysell

USA

Andrew Hill

UK

Itaru Hirai

Japan

Kenji Hirayama

Japan

Robert Hirt

UK

Cedric Hirzel

Switzerland

Michele C Hlavsa

USA

Jennifer Ho

Australia

Paulo Ho

Brazil

Nguyen Binh Hoa

Viet Nam

Marcia Hobbs

USA 


\begin{tabular}{|c|c|c|}
\hline $\begin{array}{l}\text { Natasha Hochberg } \\
\text { USA }\end{array}$ & $\begin{array}{l}\text { Chun-Ta Huang } \\
\text { Taiwan }\end{array}$ & $\begin{array}{l}\text { Daniela Huzly } \\
\text { Germany }\end{array}$ \\
\hline $\begin{array}{l}\text { Ian Hodgson } \\
\text { UK }\end{array}$ & $\begin{array}{l}\text { Huang Huang } \\
\text { USA }\end{array}$ & $\begin{array}{l}\text { Javier Ibáñez } \\
\text { Spain }\end{array}$ \\
\hline $\begin{array}{l}\text { Boris Hogema } \\
\text { The Netherlands }\end{array}$ & $\begin{array}{l}\text { Jee-Fu Huang } \\
\text { Taiwan }\end{array}$ & $\begin{array}{l}\text { Olubukola Idoko } \\
\text { Gambia }\end{array}$ \\
\hline $\begin{array}{l}\text { Celia Holland } \\
\text { Ireland }\end{array}$ & $\begin{array}{l}\text { Shiang-Fen Huang } \\
\text { Taiwan }\end{array}$ & $\begin{array}{l}\text { Vrettos Ierodiakonou } \\
\text { Greece }\end{array}$ \\
\hline $\begin{array}{l}\text { David Holland } \\
\text { USA }\end{array}$ & $\begin{array}{l}\text { Yen-Tsung Huang } \\
\text { USA }\end{array}$ & $\begin{array}{l}\text { Ricardo Igreja } \\
\text { Brazil }\end{array}$ \\
\hline $\begin{array}{l}\text { Nusrat Homaira } \\
\text { USA }\end{array}$ & $\begin{array}{l}\text { Yhu-Chering Huang } \\
\text { Taiwan }\end{array}$ & $\begin{array}{l}\text { Philip Ind } \\
\text { UK }\end{array}$ \\
\hline $\begin{array}{l}\text { Kam-Lun Ellis Hon } \\
\text { Hong Kong }\end{array}$ & $\begin{array}{l}\text { Yi-Wen Huang } \\
\text { Taiwan }\end{array}$ & $\begin{array}{l}\text { Shingo Inoue } \\
\text { Japan }\end{array}$ \\
\hline $\begin{array}{l}\text { Claire Hooker } \\
\text { Australia }\end{array}$ & $\begin{array}{l}\text { Victor Huber } \\
\text { USA }\end{array}$ & $\begin{array}{l}\text { Angela Monica Ionică } \\
\text { Romania }\end{array}$ \\
\hline $\begin{array}{l}\text { Vivian Hope } \\
\text { UK }\end{array}$ & $\begin{array}{l}\text { Mark Hudak } \\
\text { USA }\end{array}$ & $\begin{array}{l}\text { Seth Irish } \\
\text { USA }\end{array}$ \\
\hline $\begin{array}{l}\text { Phil Hopewell } \\
\text { USA }\end{array}$ & $\begin{array}{l}\text { Alan Hudson } \\
\text { USA }\end{array}$ & $\begin{array}{l}\text { Warnow Elon Isaac } \\
\text { Nigeria }\end{array}$ \\
\hline $\begin{array}{l}\text { Nobuyuki Horita } \\
\text { Japan }\end{array}$ & $\begin{array}{l}\text { Li Hui } \\
\text { China }\end{array}$ & $\begin{array}{l}\text { Nazir Ismail } \\
\text { South Africa }\end{array}$ \\
\hline $\begin{array}{l}\text { David Horn } \\
\text { USA }\end{array}$ & $\begin{array}{l}\text { Roger Hullin } \\
\text { Switzerland }\end{array}$ & $\begin{array}{l}\text { Noriko Isobe } \\
\text { USA }\end{array}$ \\
\hline $\begin{array}{l}\text { Olaf Horstick } \\
\text { Germany }\end{array}$ & $\begin{array}{l}\text { Kristina Hulten } \\
\text { USA }\end{array}$ & $\begin{array}{l}\text { Cathy Ison } \\
\text { UK }\end{array}$ \\
\hline $\begin{array}{l}\text { Catherine F Houlihan } \\
\text { UK }\end{array}$ & $\begin{array}{l}\text { Bruce Humphrey } \\
\text { UK }\end{array}$ & $\begin{array}{l}\text { Enrique Bernaola Iturbe } \\
\text { Spain }\end{array}$ \\
\hline $\begin{array}{l}\text { Thomas Allan House } \\
\text { UK }\end{array}$ & $\begin{array}{l}\text { Chao-Hung Hung } \\
\text { Taiwan }\end{array}$ & $\begin{array}{l}\text { Danielle Iuliano } \\
\text { USA }\end{array}$ \\
\hline $\begin{array}{l}\text { Thokozani Hove } \\
\text { Zimbabwe }\end{array}$ & $\begin{array}{l}\text { Ivan FN Hung } \\
\text { Hong Kong }\end{array}$ & $\begin{array}{l}\text { Martha Iwamoto } \\
\text { Uganda }\end{array}$ \\
\hline $\begin{array}{l}\text { Alison Howarth } \\
\text { UK }\end{array}$ & $\begin{array}{l}\text { Ling Lung Hung } \\
\text { Hong Kong }\end{array}$ & $\begin{array}{l}\text { Collins C Iwuji } \\
\text { UK }\end{array}$ \\
\hline $\begin{array}{l}\text { Michelle Hsiang } \\
\text { USA }\end{array}$ & $\begin{array}{l}\text { Mina Hur } \\
\text { Korea, South }\end{array}$ & $\begin{array}{l}\text { Anand Iyer } \\
\text { The Netherlands }\end{array}$ \\
\hline $\begin{array}{l}\text { Ming-Ju Hsieh } \\
\text { Taiwan }\end{array}$ & $\begin{array}{l}\text { Aeron Hurt } \\
\text { Australia }\end{array}$ & $\begin{array}{l}\text { Jacques Izopet } \\
\text { France }\end{array}$ \\
\hline $\begin{array}{l}\text { Chao Wei Hsu } \\
\text { Taiwan }\end{array}$ & $\begin{array}{l}\text { Syed Shahzad ul Hussan } \\
\text { Pakistan }\end{array}$ & $\begin{array}{l}\text { Kauser Jabeen } \\
\text { Pakistan }\end{array}$ \\
\hline $\begin{array}{l}\text { Yongfei Hu } \\
\text { China }\end{array}$ & $\begin{array}{l}\text { Wilhelmina Huston } \\
\text { Australia }\end{array}$ & $\begin{array}{l}\text { E Jacobs } \\
\text { Germany }\end{array}$ \\
\hline $\begin{array}{l}\text { Chiu-Ching Huang } \\
\text { Taiwan }\end{array}$ & $\begin{array}{l}\text { David Hutton } \\
\text { USA }\end{array}$ & $\begin{array}{l}\text { Nathalie Jacobs } \\
\text { Belgium }\end{array}$ \\
\hline
\end{tabular}


Jyotsna Jagai

USA

Amita Jain

India

Kriti Jain

USA

Hamid Jalal

UK

Vincent Jamonneau

France

Nina Jancar

Slovenia

Stephane Jauréguiberry

France

Marjan Javanbakht

USA

Deborah Jaworski

USA

Saroj Jayasinghe

Sri Lanka

Seyed Mohammad Jazayeri

Iran

Jo Jefferies

UK

Wen-Juei Jeng

Taiwan

Cheryl Jennings

USA

Anders Jensen

Denmark

Synne Jenum

Norway

Seok Hoon Jeong

South Korea

Aaron Jex

Australia

Manhong Jia

China

Judy Natalia Jiménez

Colombia

Fengyi Jin

Australia

Akanitt Jittmittraphap

Thailand
Anja Joachim

Austria

Sophia Johler

Switzerland

Reimar Johne

Germany

Matthew Johnson

USA

James Johnston

Canada

Makoto Jones

USA

Rachael Jones

Switzerland

Silje Bakken Jørgensen

Norway

Martin Joyce Brady

USA

Justin Julander

USA

Anamarija Jurcev Savicevic

Croatia

Jessica Justman

USA

Prapan Jutavijittum

Thailand

S Patrick Kachur

USA

Klaus Kaier

Germany

Siripen Kalayanarooj

Thailand

Natarajaseenivasan Kalimuthusamy India

Andrew Kambugu

Uganda

Taro Kamigaki

Japan

Witchuda Kamolvit

Thailand

Rama Kandasamy

UK

Abraham Kandathil

USA
Melissa Kang

Australia

Chuan-Liang Kao

Taiwan

Nathan Kapata

Zambia

Arti Kapil

India

Melissa Kapulu

Kenya

Beatrix Kapusinszky

USA

Drosos Karageorgopoulos

Greece

Petros Karakousis

USA

Panagiotis Karanis

Germany

Samuel Kariuki

Kenya

Symon

Kenya

Maile Karris

USA

Panduka Karunanayake

Sri Lanka

Desta Kassa

Ethiopia

Victoria Katawera

USA

Juri Katchanov

Germany

Mitsuyo Kawaguchiya

Japan

Yoshiiku Kawakami

Japan

Uner Kayabas

Turkey

Helena Käyhty

Finland

Brenda Kazemier

The Netherlands

Mary Kearney

USA 
Tibebeselassie Keflie

Ethiopia

Theodoros Kelesidis

USA

Christian Keller

Germany

David Kelso

USA

Brian Kendall

USA

Derek Kennedy

Australia

Alison Kesson

Australia

Yoav Keynan

Canada

\section{Ali Khamesipour}

Iran

Pattara Khamrin

Thailand

Muhammad Umair Khan

Malaysia

Rafiq Khanani

Pakistan

Gulam Khandaker

Australia

Ameneh Khatami

Australia

Alexander Khoruts

USA

Sandra Kik

The Netherlands

Kwang-Sik Kim

USA

Kyuseok Kim

South Korea

Hirokazu Kimura

Japan

Rachel King

Uganda

Margaret Kingston

UK

Agnes Kiragga

Uganda
Halid Kirunda

Uganda

Pandey Kishor

Nepal

Takatoshi Kitazawa

Japan

Eili Klein

USA

Karin Klingel

Germany

Hans-Michael Klinger

Germany

Eveline Klinkenberg

The Netherlands

Daniela Klobassa

Austria

Marisa Klopper

South Africa

Tejs Klug

Denmark

Jan Kluytmans

The Netherlands

Mirjam Knol

The Netherlands

Wen-Chien Ko

Taiwan

Nobumichi Kobayashi

Japan

Marleen Kock

South Africa

Sander Koenraadt

The Netherlands

Tse Hsien Koh

China

Won-Jung Koh

South Korea

Alain Kohl

UK

Gerjo Kok

The Netherlands

Olatunji Kolawole

South Africa

Marin Kollef

USA
Peter Kolominsky-Rabas

Germany

Onn Min Kon

UK

Frank Konings

Philippines

James Stephen Koopman USA

Neeltje Kootstra

The Netherlands

Izabela Korona-Glowniak

Poland

Shyam Kottilil

USA

Claudia Kozinetz

USA

John Kraemer

USA

Colleen Kraft

USA

Axel Kramer

Germany

Ellen Krautkramer

Germany

Veit Krenn

Germany

Triveni Krishnan

India

Shih-Chi Ku

Taiwan

Abhay Kudale

India

Ayush Kumar

USA

Alakes Kumar Kole

India

Mark Kuniholm

USA

Andreas Kuznil

USA

Awewura Kwara

USA

Jesse Kwiek

USA 
Kin On Kwok

Hong Kong

Laurence Lachaud

France

Philippe Lagacé-Wiens

Canada

Jennifer Lai

USA

Poh-Chin Lai

Hong Kong

Sanjay Lala

South Africa

Tommy Tsan-Yuk Lam

USA

Tracey Lamb

USA

Stephen Lambert

Australia

Mohammed Lamorde

Uganda

Ruiting Lan

Australia

Marcus D Lancé

The Netherlands

Caroline Landelle

France

Sylvia Laperche

France

Kerry LaPlante

USA

John A Larbi

Ghana

Bryce Larke

Canada

Sarah Larney

Australia

Cornelia Lass-Floerl

Austria

Eric Lau

Hong Kong

James Lawler

USA

Catherine Lawrence

UK
John Lawrenson

South Africa

Jen Layden

USA

Laura Layland

Germany

Christina Lazar

USA

Tran-Anh Le

Viet Nam

Vincent Le Moing

France

Nicole Le Saux

Canada

Bee Wah Lee

Singapore

Chun Kiat Lee

Singapore

Hong Kai Lee

Singapore

Ming-Hsun Lee

Taiwan

Sang-Won Lee

South Korea

Sue Lee

Thailand

Todd Lee

Canada

Vernon Lee

Singapore

Winnie Lee

Singapore

Jerome Legoff

France

Clara Lehmann

Germany

Sebastain Lemmen

Germany

Qibin Leng

China

Sebastiano Leone Italy

Alexander Lerner

USA
Alasdair Leslie

South Africa

Victor Leung

Canada

Peter Leutscher

Denmark

Marilyn Levi

USA

Jie Bin Lew

Australia

Karl Lewalter

Germany

David Lewis

Australia

James Lewis

USA

Joseph Lewnard

USA

George Kam Hop Li

Hong Kong

Chen-Yi Liao

Taiwan

Qiuyan Liao

Hong Kong

Wanqing Liao

China

Yun-Fan Liaw

Taiwan

Patrick Lillie

UK

Jason Limberis

South Africa

Wolfgang Lindner

Germany

Filippo Lipani

Italy

Christopher Lippincott

USA

Thiago Lisboa

Spain

Margaret Littlejohn

Australia

Feng Liu

USA 
Hsin-Fu Liu

Taiwan

Jie Liu

USA

Ming-Tsan Liu

Taiwan

Sze Hang Kevin Liu

Hong Kong

Vivian Liu

China

Wei Liu

China

Martin Llewellyn

UK

Michael Lo

USA

Catherine M Loc-Carrillo

USA

Nicoletta Locuratolo

Italy

Tze Ping Loh

Singapore

Elisa Long

USA

Richard Long

Canada

Yves Longtin

Canada

Ana Patricia Lopes

Portugal

Guillermo Lopez Campos

Australia

Luis Eduardo López-Cortés

Spain

Ben Lopman

UK

Irene Lorand-Metze

Brazil

Jaime Lora-Tamayo

Spain

Pierre Loulergue

France

Christopher Lowe

Canada
Warren Lowman

South Africa

Beibei Lu

USA

Po-Liang Lu

Taiwan

Vivian Luchsinger

Chile

Christoph Luebbert

Germany

Leo Lui Hong

Kong

Robert Lukande

Uganda

Aroonlug Lulitanond

Thailand

David Lung

Hong Kong

Christian Lüring

Germany

Irja Lutsar

Estonia

Philipp Lutz

Germany

Zhiyue Lv

China

Dickson Lwetoijera

Tanzania

Samantha Lycett

UK

David Chien Lye

Singapore

G Marshall Lyon

USA

Gary Maartens

South Africa

Dorothy Machalek

Australia

Shingai Machingaidze

South Africa

David MacLaren

Australia

Gesham Magombedze

UK
FMG Magpantay

USA

Jagadish Mahanta

India

Mohammad Mahmoudi

Iran

Matthias Maiwald

Germany

Gathsaurie Malavige

Sri Lanka

Maricar Malinis

USA

Iwona Malinowska

Poland

Kylie-Ann Mallitt

Australia

Ryan Malosh

USA

Monica Malta

USA

Helen Maltezou

Greece

Caterina Mammina

Italy

Si Ming Man

USA

Ogenna Manafa

Ireland

Niwat Maneekarn

Thailand

Jose Ramon Maneiro

Spain

Julie Mangino

USA

Lisa Manhart

USA

Jayanti Mania-Pramanik India

Tobias Manigold

Switzerland

Annette Mankertz

Germany

Jennifer Manne

USA 
Oriol Manuel

Switzerland

Lamberto Manzoli

Italy

Suresh Maray

Saudi Arabia

Giulia Marchetti

Italy

Andres Mardh

Sweden

Jutta Marfurt

Australia

Alemka Markotic

Croatia

Theodore Marras

Canada

Carl Marrs

USA

Thomas Marth

Germany

Emily Martin

USA

Irene Martin

Canada

Javier Martin

UK

Maria Martin

Germany

Richard A Martinello

USA

Esteban Martinez

Spain

Ignacio Martin-Loeches

Ireland

Florian Marx

Germany

Emilio Maseda

Spain

Mar Masiá

Spain

Paul Massion

Belgium

Serge Masson

Italy
Cesare Massone

USA

Claudio Maria

Mastroianni Italy

Amy Mathers

USA

Catherine Mathews

UK

Yasufumi Matsumura

Japan

Alberto Matteelli

Italy

Dimitrios Matthaiou

Greece

Frauke Mattner

Germany

Eric Maury

France

Harriet Mayanja

Uganda

Humphrey Mazigo

Tanzania

Wilfred Mbacham

Cameroon

Cecilia Mbae

Kenya

Anthony Mbonye

Uganda

Erasto Vitus Mbugi

Tanzania

Sheryl McCurdy

USA

Scott McDonald

The Netherlands

Fiona McGill

UK

Rose McGready

Thailand

Skye McGregor

Australia

Bradford McGwire

USA

Dorian McIlroy

France
Mark McKinlay

USA

Mary-Louise McLaws

Australia

Diane McMahon-Pratt

USA

Jim McMenamin

UK

Meredith McMorrow

USA

Brendan McMullan

Australia

Takafira Mduluza

Zimbabwe

Jaishri Mehraj

Germany

Sanjay Mehta

USA

Roberto Melano

Canada

Analy Melo

Brazil

Francesco Menichetti

Italy

Shruti Menon

Australia

Andreas Mentis

Greece

Andres Merits

Estonia

Sharon Meropol

USA

Dominik Mertz

Canada

Jane Messina

UK

David Meya

Uganda

Jaimie Meyer

USA

Patrick Miailhes

France

Janet Midega

Kenya 
Florence Migot-Nabias

France

Benjamin Miko

USA

Rafael T Mikolajczyk

Germany

Michael Millar

UK

Ruth Miller

Canada

Richard Milne

New Zealand

Cezarina Mindru

USA

Corrado Minetti

UK

Veenu Minhas

USA

Victor Minichiello

Australia

Anabela Miranda

Portugal

Angelica Miranda

Brazil

Veriko Mirtskhulava

Georgia

Sharmistha Mishra

Canada

Evangelos Misiakos

Greece

Carole Mitnick

USA

Masashi Mizuguchi

Japan

Hala Mohamed

Egypt

Vidyarani Mohankumar

India

Igor Mokrousov

Russia

Monica Molano

Australia

Edmund Molesworth

Australia
Dinesh Mondal

Bangladesh

Francesca Montagnani

Italy

Brian Montague

USA

Matilde Monteiro-Soares

Portugal

Victor Monteon

Mexico

Christopher Montgomery

USA

Giovanni Montini

Italy

Luke Moore

UK

Roger Morbey

UK

Chantal Morel

UK

Frederick Morfaw

Cameroon

Mitsuru Mori

Japan

Fintan Moriarty

Switzerland

Hiroyuki Moriuchi

Japan

Carl Morrow

South Africa

Joel Mossong

Luxembourg

Domenico Motola

Italy

Moustafa Mourad

UK

Sikhulile Moyo

Botswana

Sizulu Moyo

South Africa

Sekesai Mtapuri-Zinyowera

Zimbabwe

Eric Mueller

USA
Lapo Mughini-Gras

The Netherlands

Vikramjit Mukherjee

USA

JK Mukonzo

Sweden

Christiaan Mulder

The Netherlands

Matthew Muller

Canada

Mathirut Mungthin

Thailand

Carmen Munoz-Almagro

Spain

David Murdoch

New Zealand

Oscar Murillo

Spain

Sean Murphy

USA

Baba Maiyaki Musa

Nigeria

John Muscedere

Canada

David Mushatt

USA

Doris Mutabazi-Mwesigire

Uganda

Nico Mutters

Germany

Bernard Naafs

The Netherlands

Kesara Na-Bangchang

Thailand

Jean Nachega

USA

Mathieu Nacher

French Guiana

Behzad Nadjm

Viet Nam

Y Nagao

Japan

Nico Nagelkerke

The Netherlands 


\begin{tabular}{|c|c|c|}
\hline $\begin{array}{l}\text { M Nagl } \\
\text { Austria }\end{array}$ & $\begin{array}{l}\text { Peter Neal } \\
\text { UK }\end{array}$ & $\begin{array}{l}\text { Ran Nir-Paz } \\
\text { Israel }\end{array}$ \\
\hline Cho Naing & Issa Nebie & Veeranoot Nissapatorn \\
\hline Malaysia & Burkina Faso & Malaysia \\
\hline Vidhya Nair & Kenrad Nelson & Rashed Noor \\
\hline Canada & USA & Bangladesh \\
\hline Vinay Nair & Mark Nelson & Ingvild Nordøy \\
\hline USA & UK & Norway \\
\hline Luigi Naldi & Alexander Nemec & Hans Norrgren \\
\hline Italy & Czech Republic & Sweden \\
\hline Lee Nan-Yao & Ujjwal Neogi & Steven Norris \\
\hline Taiwan & Sweden & USA \\
\hline Kanwar Narain & Hui-Min Neoh & Abigail Norris Turner \\
\hline India & Malaysia & USA \\
\hline Edward Nardell & Heinrich Neubauer & Niels Nørskov \\
\hline USA & Germany & Denmark \\
\hline Giuseppe Nascetti & Jason Newland & Monika Nothacker \\
\hline Italy & USA & Germany \\
\hline Ana Lucia Nascimento & Kim Tien Ng & Shannon Novosad \\
\hline Brazil & Malaysia & USA \\
\hline Cristiana Nascimento-Carvalho & Mah-Lee Ng & Norbert Nowotny \\
\hline Brazil & Singapore & Austria \\
\hline K Natarajaseenivasan & Nadia Nguyen & Saad Nseir \\
\hline India & USA & France \\
\hline Brian Nathanson & Mya Myat Ngwe Tun & Elaine Nsoesie \\
\hline USA & Japan & USA \\
\hline Sonia Navas-Martin & Mancini Nicasio & Melissa Nyendak \\
\hline USA & Italy & USA \\
\hline Seema Nayak & Christos Nicolaides & Ali O Mohamed Salem O Boukhary \\
\hline USA & USA & Mauritania \\
\hline Godwin Nchinda & Lindsay Nicolle & Michael Oberholzer \\
\hline Cameroon & Canada & USA \\
\hline Momar Ndao & Matthias Niedrig & Karina O'Connell \\
\hline Canada & Germany & Ireland \\
\hline Martial Ndeffo Mbah & Susanne Dam Nielsen & Catherine O'Connor \\
\hline USA & Denmark & Australia \\
\hline Dieynaba N'Diaye & Birgit Nikolay & Max O'Donnell \\
\hline France & UK & USA \\
\hline James Neal & Hans Helmut Niller & Onyema Ogbuagu \\
\hline UK & Germany & USA \\
\hline Jay Neal & Chuanyi Ning & Sung Hee Oh \\
\hline USA & China & South Korea \\
\hline Keith Neal & Yuming Ning & Makoto Ohnishi \\
\hline UK & USA & Japan \\
\hline
\end{tabular}




\begin{tabular}{|c|c|c|}
\hline $\begin{array}{l}\text { John Ohoro } \\
\text { USA }\end{array}$ & $\begin{array}{l}\text { Carla Osiowy } \\
\text { Canada }\end{array}$ & $\begin{array}{l}\text { Lucia Pallecchi } \\
\text { Italy }\end{array}$ \\
\hline Iruka Okeke & Fabio Ostanello & Kelli Palmer \\
\hline Nigeria & Italy & USA \\
\hline $\begin{array}{l}\text { Lucy Okell } \\
\text { UK }\end{array}$ & $\begin{array}{l}\text { Ronan O'Toole } \\
\text { Australia }\end{array}$ & $\begin{array}{l}\text { Domingo Palmero } \\
\text { Argentina }\end{array}$ \\
\hline $\begin{array}{l}\text { Sinan Oksuz } \\
\text { Turkey }\end{array}$ & $\begin{array}{l}\text { Akaninyene Otu } \\
\text { Nigeria }\end{array}$ & $\begin{array}{l}\text { Stefan Panaiotov } \\
\text { Bulgaria }\end{array}$ \\
\hline $\begin{array}{l}\text { Afiong Oku } \\
\text { Nigeria }\end{array}$ & $\begin{array}{l}\text { Kennedy Otwombe } \\
\text { South Africa }\end{array}$ & $\begin{array}{l}\text { Aditya K Panda } \\
\text { India }\end{array}$ \\
\hline $\begin{array}{l}\text { Kehinde Okunade } \\
\text { Nigeria }\end{array}$ & $\begin{array}{l}\text { Mohamed Salem Ould Ahmedou Salem } \\
\text { Mauritania }\end{array}$ & $\begin{array}{l}\text { Xiao-Li Pang } \\
\text { Canada }\end{array}$ \\
\hline $\begin{array}{l}\text { Sam Okware } \\
\text { Uganda }\end{array}$ & $\begin{array}{l}\text { Collins Ouma } \\
\text { Kenya }\end{array}$ & $\begin{array}{l}\text { Annalisa Pantosti } \\
\text { Italy }\end{array}$ \\
\hline $\begin{array}{l}\text { Anna Olsen } \\
\text { Australia }\end{array}$ & $\begin{array}{l}\text { Alexander Outhred } \\
\text { Australia }\end{array}$ & $\begin{array}{l}\text { Pasquali Paolo } \\
\text { Italy }\end{array}$ \\
\hline $\begin{array}{l}\text { Karina Olsen } \\
\text { Norway }\end{array}$ & $\begin{array}{l}\text { Helieh Oz } \\
\text { USA }\end{array}$ & $\begin{array}{l}\text { Vassiliki Papaevangelou } \\
\text { Greece }\end{array}$ \\
\hline $\begin{array}{l}\text { Yusuf Omosun } \\
\text { USA }\end{array}$ & $\begin{array}{l}\text { S Ozkoc } \\
\text { Turkey }\end{array}$ & $\begin{array}{l}\text { Jesse Papenburg } \\
\text { Canada }\end{array}$ \\
\hline $\begin{array}{l}\text { Geoffrey Omuse } \\
\text { Kenya }\end{array}$ & $\begin{array}{l}\text { Marc Paccalin } \\
\text { France }\end{array}$ & $\begin{array}{l}\text { Manuela Papini } \\
\text { Italy }\end{array}$ \\
\hline $\begin{array}{l}\text { Raphael Ondondo } \\
\text { Kenya }\end{array}$ & $\begin{array}{l}\text { Maria Clara Padoveze } \\
\text { Brazil }\end{array}$ & $\begin{array}{l}\text { Glaucia Paranhos-Baccalà } \\
\text { France }\end{array}$ \\
\hline $\begin{array}{l}\text { Jason Ong } \\
\text { Australia }\end{array}$ & $\begin{array}{l}\text { Anne-Laure Page } \\
\text { France }\end{array}$ & $\begin{array}{l}\text { Ramesh Paranjape } \\
\text { India }\end{array}$ \\
\hline $\begin{array}{l}\text { Gabriel Mbadiwe Onyeama } \\
\text { Nigeria }\end{array}$ & $\begin{array}{l}\text { Katie Page } \\
\text { Australia }\end{array}$ & $\begin{array}{l}\text { Dimitrios Paraskevis } \\
\text { Greece }\end{array}$ \\
\hline $\begin{array}{l}\text { Eline Op De Coul } \\
\text { The Netherlands }\end{array}$ & $\begin{array}{l}\text { John Paget } \\
\text { The Netherlands }\end{array}$ & $\begin{array}{l}\text { Raveen Parboosing } \\
\text { South Africa }\end{array}$ \\
\hline $\begin{array}{l}\text { Paula Opromolla } \\
\text { Brazil }\end{array}$ & $\begin{array}{l}\text { W Pai } \\
\text { USA }\end{array}$ & $\begin{array}{l}\text { Christopher Parry } \\
\text { UK }\end{array}$ \\
\hline $\begin{array}{l}\text { Eyal Oren } \\
\text { USA }\end{array}$ & $\begin{array}{l}\text { Elijah Paintsil } \\
\text { USA }\end{array}$ & $\begin{array}{l}\text { Fiona Parsonson } \\
\text { Australia }\end{array}$ \\
\hline $\begin{array}{l}\text { Walter Orenstein } \\
\text { USA }\end{array}$ & $\begin{array}{l}\text { Dasja Pajkrt } \\
\text { The Netherlands }\end{array}$ & $\begin{array}{l}\text { Shama Parveen } \\
\text { India }\end{array}$ \\
\hline $\begin{array}{l}\text { Thorsten Orlikowsky } \\
\text { Germany }\end{array}$ & $\begin{array}{l}\text { Andrea Pakula } \\
\text { USA }\end{array}$ & $\begin{array}{l}\text { Jotam Pasipanodya } \\
\text { USA }\end{array}$ \\
\hline $\begin{array}{l}\text { Beatrix Oroszi } \\
\text { Hungary }\end{array}$ & $\begin{array}{l}\text { Elizabeth Palavecino } \\
\text { USA }\end{array}$ & $\begin{array}{l}\text { Tushar B Patil } \\
\text { India }\end{array}$ \\
\hline $\begin{array}{l}\text { Giovanni Battista Orsi } \\
\text { Italy }\end{array}$ & $\begin{array}{l}\text { Antonio Palazon-Bru } \\
\text { Spain }\end{array}$ & $\begin{array}{l}\text { John Paul } \\
\text { UK }\end{array}$ \\
\hline $\begin{array}{l}\text { Enrique Ortega-Gonzalez } \\
\text { Spain }\end{array}$ & $\begin{array}{l}\text { Pedro Palha } \\
\text { Brazil }\end{array}$ & $\begin{array}{l}\text { Nicole Pavio } \\
\text { France }\end{array}$ \\
\hline
\end{tabular}


Androula Pavli

Greece

Eric Walter Pefura-Yone

Cameroon

Ville Peltola

Finland

Ruirui Peng

China

\section{Adriana Perez}

USA

Steven Pergam

USA

Guey Chuen Perng

Taiwan

Kristina Persson

Sweden

Vivekanandan Perumal

India

Jonathan Peter

South Africa

Linda Petrone

Italy

Mariya Petrova

Belgium

Michael Pfaller

USA

Yvonne Pfeifer

Germany

Jody Phelan

UK

Warren Phipps

USA

Juan Picazo

Spain

Russel Pierre

Jamaica

Antonio Pignatari

Brazil

Benoît Pilmis

France

Marcelo Pinto

Brazil

Lionel Piroth

France
Henry Pleass

Australia

Ioannis Pneumatikos

Greece

Laura Jean Podewils

USA

Jason Pogue

USA

Piero Poletti

Italy

Chiara Poletto

France

Kevin Pollock

UK

Larisa Poluektova

USA

Virginia Pomar

Spain

William Pomat

Guinea

Kittiyod Poovorawan

Thailand

Isabel Portugal

Portugal

Luciano Potena

Italy

Lakshmi-Prasad Potluri

USA

Stephanie Pouch

USA

Anja Poulsen

Denmark

Spyros Pournaras

Greece

Gerard Pouvourville

USA

Koen B Pouwels

The Netherlands

Isobel Poynten

Australia

Bruno Pozzetto

France

Prabda Praphasiri

Thailand
Rosa Prato

Italy

George Praygod

Tanzania

Rebecca Prevots

USA

Patricia Priest

New Zealand

Miroslav Prucha

Czech Republic

Bhupesh Prusty

Denmark

Maria Carmen Puertas

Spain

Chengli Que

China

Mark Quinlivan

UK

Michael Quinn

Australia

Miguel Quinones-Mateu

USA

Yvonne Qvarnstrom

USA

Tavs Qvist

Denmark

R Negroni

Argentina

R Prakash

India

Holger F Rabenau

Germany

Keith Radcliffe

UK

Lewis Radonovich

USA

BE Rangaswamy

India

Gilles Raguin

France

Jaya Rajaiya

USA

Anna Ralph

Australia 
Sanjay Ram

USA

Girish Ramachandran

USA

Oscar Ramirez

USA

Jose Manuel Ramos

Spain

Jake Rance

Australia

Louise Randall

Australia

Tara Randis

USA

Merja Rantala

Finland

Durga Rao

India

Didier Raoult

France

Jean-Philippe Rasigade

France

Abdolaziz Rastegar Lari

Iran

Mehrdad Ravanshad

Iran

Henrik Ravn

Denmark

G Thomas Ray

USA

Pallab Ray

India

Jean-Baptiste Rayaisse

Burkina Faso

Michael Rayment

UK

Phillip Read

Australia

Tim Read

Australia

Timothy Read

USA

Patrick Reading

Australia
Gil Redelman-Sidi

USA

David Regan

Australia

Thomas Reiberger

Austria

Utz Reichard

Germany

Keli Cristine Reiter

Brazil

Lili Ren

China

Pilar Retamar

Spain

Kelly Reveles

USA

Paula Revell

USA

Heidi Reynolds

USA

Nancy Reynolds

USA

Joshua Rhein

USA

Peter Rice

USA

Vincent Richard

Senegal

Riina Richardson

UK

Jan Hendrik Richardus

New Zealand

Sara Richter

Italy

Michaela Riddell

Australia

David Riedel

USA

Eirini Rigopoulou

Greece

Julien Riou

France

Ariel Rivas

USA
Antonio Rivero

Spain

Laura Rivino

Singapore

Marco Rizzi

Italy

Jennifer Roberts

Australia

Rachel Robinson

USA

Monica Rocco

Italy

Igor Rodrigues

Brazil

Sabrina Rodriguez-Campos

Switzerland

Benjamin Rogers

Australia

Gabriel Rojas-Ponce

Peru

Thierry Rolling

UK

Nigel Rollins

South Africa

William Rom

UK

Anne Marie Roque-Afonso

France

Nicolas Rose

France

Joshua Ross

Australia

Sarah Rowland-Jones

UK

Patricia Ruas-Madiedo

Spain

Franco Maria Ruggeri

Italy

Diego Ruiz Moreno

USA

Heiner Ruschulte

Germany

Fiona Russell

Australia 
George Rutherford

USA

Eva Ruzic-Sabljic

Slovenia

Magda Rybicka

Poland

Shiva S Halli

Canada

Saraswathy Sabanathan

Viet Nam

Francesca Sabbatini

Italy

Carla Sabia

Italy

Caroline Sabin

UK

Lora Sabin

USA

Karuna Sagili

India

Bibhuti Saha

India

Jitendra Sahu

India

Akihiko Saitoh

Japan

Eduardo Salazar-Lindo

Peru

Max Salfinger

USA

Cassandra Salgado

USA

Faouzy Saliba

France

Fernando Salvador

Spain

Nadia Sam-Agudu

Nigeria

Taraz Samandari

USA

Banu Sancak

Turkey

Susan Sanchez

USA
Alexandra Sánchez

Brazil

Naveen Sankhyan

India

Pitak Santanirand

Thailand

Cristina Santos

Portugal

Daniel Santos

Brazil

Jose Ramon Santos

Spain

Roberto Santos

USA

Rajiv Sarkar

India

Bahador Sarkari

Iran

Manoj Sarma

USA

Joe Sasadeusz

Australia

Michael Satlin

USA

Sarah Satola

USA

Catherine Satzke

Australia

Andreas Sauerbrei

Germany

John Saunders

UK

Frank Scannapieco

USA

Simon Schaaf

South Africa

Frieder Schaumburg

Germany

Simone Scheithauer

Germany

Andre Scherag

Germany

Consuelo Schiroli

Italy
Susanne Schmitz

Ireland

Kathryn Schnippel

South Africa

Henrik Carl Schønheyder

Denmark

Pieter Schreuder

The Netherlands

Constance Schultsz

The Netherlands

Lucas Schulz

USA

Jörg Schüpbach

Switzerland

Andrew Schwaderer

USA

William Schwan

USA

Jeremy Schwartz

USA

John Scott

USA

Eric Seaberg

USA

Andrew Seaton

UK

Ludwig Sedlacek

Germany

Gad Segal

Israel

Edelwisa Segubre-Mercado

Philippines

Marva Seifert

USA

Moorine Penninah Sekadde

Uganda

Aggrey Semeere

Uganda

Joseph Sempa

Uganda

Chaminda Jayampath Seneviratne Singapore

Piseth Seng

France 
Elisavet Serti

USA

Chetan Seshadri

USA

Ajay Sethi

USA

Alberto Severini

Canada

Fiona Shackley

UK

Lena Shah

Canada

Nipam Shah

USA

Shohreh Shahmahmoodi

Iran

Laura Shallcross

UK

Isdore Chola Shamputa

Canada

Masoomeh Shams-Ghahfarokhi

Iran

Arun Sharma

India

Pragya Sharma

USA

Kirill Sharshov

Russia

Kimberly Shea

USA

Xuzhuang Shen

China

Edward Sherwood

USA

Veena Shetty

India

Jim Sheu

Taiwan

Marcia Shew

USA

Hiroyuki Shimizu

Japan

Sourya Shrestha

USA
Chin-Chung Shu

Taiwan

P Shuetz

Switzerland

Dhaval Shukla

India

Jennifer Shuldiner

Israel

Emily Sickbert-Bennett

USA

Scott Sieg

USA

Ilias Siempos

Greece

Sheetal Silal

South Africa

Nozza Silvia

Italy

Bryony Simmons

UK

Victoria Simms

UK

Patricia Simner

USA

Itu Singh

India

Leung Kei Siu

Taiwan

Girts Skenders

Latvia

Donald Skinner

South Africa

Tina Skinner-Adams

Australia

Lars Skog

Sweden

David Skurnik

USA

Miha Skvarc

Slovenia

Emma Slaymaker

UK

Robert Slinger

Canada
Derek Sloan

Malawi

Hans-Christian Slotved

Denmark

Colette Smit

The Netherlands

Anja Smith

South Africa

Kellie Smith

USA

Stephen Smith

Ireland

Alex Smithson

Spain

Davida Smyth

USA

René Snacken

Sweden

Li-Hwei Sng

Singapore

Evan Snitkin

USA

Kathryn Snow

Australia

Nidhi Sofat

UK

RS Solomons

Zambia

Mark Sonderup

South Africa

Manish Soneja

India

Elijah Songok

Kenya

Geoffrey Sonn

USA

Pam Sonnenberg

UK

Geeta Sood

USA

Seema Sood

India

Antoni Soriano-Arandes Spain 
Timo Sorsa

Finland

Nestor Sosa

Panama

Ana E Sousa

Portugal

Neila Souza

Brazil

D Sow

France

Vincenzo Spagnuolo

Italy

Lodewijk Spanjaard

The Netherlands

Iris Spiliopoulou

Greece

Sandra Springer

USA

Nicola Squillace

Italy

Chandrashekhar Sreeramareddy

Nepal

Saranya Sridhar

UK

Willy Ssengooba

Uganda

Janneke Stalenhoef

The Netherlands

Cecilia Stålsby Lundborg

Sweden

Lola Stamm

USA

Carol Stanciu

Romania

Miles Stanford

UK

Catherine Stein

USA

Ellen Stein

USA

Mart L Stein

The Netherlands

Joerg Steinmann

Germany
Peter Steinmann

Switzerland

Niamh Stephenson

Australia

Kasia Stepniewska

UK

Erin Stern

South Africa

Vanessa Stevens

USA

Andrew J Stewardson

Australia

Benoit Stijlemans

USA

Ellen Stobberingh

The Netherlands

Nicole Stoesser

UK

Jason Stout

USA

Kristoffer Strålin

Sweden

Andrea Streng

Germany

Klemen Strle

USA

Birgit Strommenger

Germany

Carol Strong

Taiwan

Judith Strymish

USA

Chien-Wei Su

Taiwan

Arnold Suda

Germany

Staci Sudenga

USA

Jonathan D Sugimoto

USA

Barbara Suligoi

Italy

Giorgia Sulis

Italy
David Sullivan

USA

Ayako Sumi

Japan

Ping Sun

USA

Yan Sun

Singapore

Lavanya Suneetha India

Michael Surette

Canada

Johari Surin

Malaysia

Faisal Syed

USA

Piotr Szweda

Poland

Alexis Tabah

Australia

Saadia Tabassum

Pakistan

Sepehr Tabrizi

Australia

Terence Tafatatha

UK

Patrick Taffé

Switzerland

Sarah Taimur

USA

Ai Takano

Japan

Antoine Talarmin

France

Banhock Tan

Singapore

Kit Mun Tan

Malaysia

Litjen (LJ) Tan

USA

Thuan-Tong Tan

Singapore

Tina Tan

USA 


\begin{tabular}{|c|c|c|}
\hline $\begin{array}{l}\text { Atsuchi Tanaka } \\
\text { Japan }\end{array}$ & $\begin{array}{l}\text { Eyasu Teshale } \\
\text { USA }\end{array}$ & $\begin{array}{l}\text { Egídio Torrado } \\
\text { Portugal }\end{array}$ \\
\hline $\begin{array}{l}\text { Mariza Tancredi } \\
\text { Brazil }\end{array}$ & $\begin{array}{l}\text { Raymond Tetteh } \\
\text { Ghana }\end{array}$ & $\begin{array}{l}\text { Francesco Torre } \\
\text { Italy }\end{array}$ \\
\hline $\begin{array}{l}\text { Aaron Tande } \\
\text { USA }\end{array}$ & $\begin{array}{l}\text { Elli Theel } \\
\text { USA }\end{array}$ & $\begin{array}{l}\text { Antoni Torres } \\
\text { Spain }\end{array}$ \\
\hline $\begin{array}{l}\text { Julian Wei Tze Tang } \\
\text { UK }\end{array}$ & $\begin{array}{l}\text { Robert Thimme } \\
\text { Germany }\end{array}$ & $\begin{array}{l}\text { Anna Maria Tortorano } \\
\text { Italy }\end{array}$ \\
\hline $\begin{array}{l}\text { Min Moon Tang } \\
\text { Malaysia }\end{array}$ & $\begin{array}{l}\text { Matthew Thoendel } \\
\text { USA }\end{array}$ & $\begin{array}{l}\text { Grazia Tosone } \\
\text { Italy }\end{array}$ \\
\hline $\begin{array}{l}\text { Weiming Tang } \\
\text { China }\end{array}$ & $\begin{array}{l}\text { Corinne Thompson } \\
\text { Viet Nam }\end{array}$ & $\begin{array}{l}\text { Patricia Totten } \\
\text { UK }\end{array}$ \\
\hline $\begin{array}{l}\text { Weiming Tang } \\
\text { USA }\end{array}$ & $\begin{array}{l}\text { Kimberly Thompson } \\
\text { USA }\end{array}$ & $\begin{array}{l}\text { Chafia Touil-Boukoffa } \\
\text { Algeria }\end{array}$ \\
\hline $\begin{array}{l}\text { Yi-Wei Tang } \\
\text { USA }\end{array}$ & $\begin{array}{l}\text { Emily Thorell } \\
\text { USA }\end{array}$ & $\begin{array}{l}\text { Katy Town } \\
\text { UK }\end{array}$ \\
\hline $\begin{array}{l}\text { Meltem Tasbakan } \\
\text { Turkey }\end{array}$ & $\begin{array}{l}\text { Guy Thwaites } \\
\text { Viet Nam }\end{array}$ & $\begin{array}{l}\text { Andrea Tramarin } \\
\text { Italy }\end{array}$ \\
\hline $\begin{array}{l}\text { Carlo Tascini } \\
\text { Italy }\end{array}$ & $\begin{array}{l}\text { Huai-Yu Tian } \\
\text { China }\end{array}$ & $\begin{array}{l}\text { Barbara Trautner } \\
\text { USA }\end{array}$ \\
\hline $\begin{array}{l}\text { Nancy Tee } \\
\text { Singapore }\end{array}$ & $\begin{array}{l}\text { Ellen Tijsse-Klasen } \\
\text { The Netherlands }\end{array}$ & $\begin{array}{l}\text { Elina Trembizki } \\
\text { Australia }\end{array}$ \\
\hline $\begin{array}{l}\text { Felipe Teixeira De Mello Freitas } \\
\text { Brazil }\end{array}$ & $\begin{array}{l}\text { Marcello Tirani } \\
\text { Italy }\end{array}$ & $\begin{array}{l}\text { Mariangela Trindade } \\
\text { Brazil }\end{array}$ \\
\hline $\begin{array}{l}\text { Fasil Tekola-Ayele } \\
\text { USA }\end{array}$ & $\begin{array}{l}\text { Saruda Tiwananthagorn } \\
\text { Thailand }\end{array}$ & $\begin{array}{l}\text { Quynh Mai Trinh } \\
\text { Viet Nam }\end{array}$ \\
\hline $\begin{array}{l}\text { Raymond Tellier } \\
\text { Canada }\end{array}$ & $\begin{array}{l}\text { Dileep Tiwari } \\
\text { South Africa }\end{array}$ & $\begin{array}{l}\text { Birger Trollfors } \\
\text { Sweden }\end{array}$ \\
\hline $\begin{array}{l}\text { John Tembo } \\
\text { China }\end{array}$ & $\begin{array}{l}\text { Michele Tizzoni } \\
\text { Italy }\end{array}$ & $\begin{array}{l}\text { Marius Trøseid } \\
\text { Norway }\end{array}$ \\
\hline $\begin{array}{l}\text { David Templeton } \\
\text { Australia }\end{array}$ & $\begin{array}{l}\text { Kelvin To } \\
\text { Hong Kong }\end{array}$ & $\begin{array}{l}\text { Stephanie Troy } \\
\text { USA }\end{array}$ \\
\hline $\begin{array}{l}\text { Gabriel Tender } \\
\text { USA }\end{array}$ & $\begin{array}{l}\text { Nguyen Linh Toan } \\
\text { Viet Nam }\end{array}$ & $\begin{array}{l}\text { Allan Truant } \\
\text { USA }\end{array}$ \\
\hline $\begin{array}{l}\text { Christine Teng } \\
\text { Singapore }\end{array}$ & $\begin{array}{l}\text { Selma Tobudic } \\
\text { Austria }\end{array}$ & $\begin{array}{l}\text { Cecilia Trucchi } \\
\text { Italy }\end{array}$ \\
\hline $\begin{array}{l}\text { Masanori Terajima } \\
\text { USA }\end{array}$ & $\begin{array}{l}\text { Viktorija Tomic } \\
\text { Slovenia }\end{array}$ & $\begin{array}{l}\text { Feng-Jen Tsai } \\
\text { Taiwan }\end{array}$ \\
\hline $\begin{array}{l}\text { Dianne (Anja) Terlouw } \\
\text { UK }\end{array}$ & $\begin{array}{l}\text { Steven Tong } \\
\text { Australia }\end{array}$ & $\begin{array}{l}\text { Eiirni Tsakiridou } \\
\text { Greece }\end{array}$ \\
\hline $\begin{array}{l}\text { Anders Ternhag } \\
\text { Sweden }\end{array}$ & $\begin{array}{l}\text { Estee Torok } \\
\text { USA }\end{array}$ & $\begin{array}{l}\text { Herman Tse } \\
\text { Hong Kong }\end{array}$ \\
\hline $\begin{array}{l}\text { Vanessa Terra } \\
\text { UK }\end{array}$ & $\begin{array}{l}\text { Kwasi Torpey } \\
\text { Nigeria }\end{array}$ & $\begin{array}{l}\text { Wondewosen Tsegaye } \\
\text { Ethiopia }\end{array}$ \\
\hline
\end{tabular}


Kuo-Chih Tseng

Taiwan, Republic Of China

Maria Tsolia

Greece

Vivien Tsu

USA

Edouard Tuaillon

France

Joseph Tucker

China

Susan Tuddenham
USA

Carrie Tudor

USA

Christine Turenne

Canada

Joanne Turner

USA

Paul Turner

Cambodia

Robin Turner

Australia

Ombretta Turriziani

Italy

Jimmy Twin

Australia

Shahadat Uddin

Australia

Kingsley Nnanna Ukwaja

Nigeria

Noman Ul Haq

Pakistan

Kristen Underhill

USA

AM Upfill-Brown

USA

Vytautas Usonis

Lithuania

Louis Valiquette

Canada

Andrew Vallely

Australia

Aarthy Vallur

USA
Florent Valour

France

Marita van de Laar

USA

Gerd van der Auwera

Belgium

Menno van der Eerden

The Netherlands

Tonja van der Kuyl

The Netherlands

Mark van der Linden

Germany

Nicoline van der Maas

The Netherlands

Marianne $A B$ van der Sande

The Netherlands

GD van der Spuy

South Africa

Martie van der Walt

South Africa

H Rogier van Doorn

Viet Nam

David van Duin

USA

AM van Furth

The Netherlands

Caroline van Gemert

Australia

Martijn van Griensven

Germany

Nelda van Soelen

South Africa

Ronald van Toorn

South Africa

G Van Well

The Netherlands

Bias Vandack

Brazil

Dana Vanlandingham

USA

Anna $\mathrm{H}$ van't $\mathbf{H o o g}$

The Netherlands

Gilles Vanwalleghem

Belgium
Sophie Vanwambeke

Belgium

Anne Vardo-Zalik

USA

Daniel Vitor Vasconcelos Santos

Brazil

Vipin M Vashishtha

India

Shawn Vasoo

Singapore

María Velasco

Spain

Mario Venditti

Italy

Delphis Vera

Peru

$S$ Vergara

Spain

Paul Verhoeven

France

Neena Verma

India

Eva Veronesi

Switzerland

Florens GA Versteegh

The Netherlands

Evelien Verstraete

Belgium

Suzanne Verver

The Netherlands

Jaco J Verweij

The Netherlands

Frédéric Veyrier

Canada

Diego Viasus

Colombia

David Vickers

Canada

Ivan Vickers

Jamaica

Roberto Vidal

Chile

Margarida Vigário

Portugal 
Tara Vijayan

USA

Dhanasekaran Vijaykrishna

Singapore

Miguel Vinas

Spain

Manfrin Vinicio

Italy

Christopher Vinnard

USA

DH Visser

The Netherlands

Marianne Visser

South Africa

Matteo Vitali

Italy

Lenka Vodstrcil

Australia

Martin Vogel

Germany

Antonio Volpi

Italy

Andrea Von Groll

Brazil

Claire Von Mollendorf

South Africa

Lutz Von Müller

Germany

Ann Vossen

The Netherlands

Lung Vu

USA

Tytti Vuorinen

Finland

Jeannette Wadula

South Africa

Florian Martin Erich Wagenlehner

Germany

Jesse Waggoner

USA

Alpana Waghmare

USA

Helga Wagner

Austria
Catriona Waitt

UK

Tim Walker

Rwanda

Elisabetta Walters

South Africa

Jann-Tay Wang

Taiwan

Jann-Yuan Wang

Taiwan

Jinfeng Wang

China

Lin Wang

Taiwan

Maggie Haitian Wang

Hong Kong

Sheng-Fan Wang

Taiwan

Tao Wang

China

Deborah Ward

UK

Nicola Wardrop

UK

David Wareham

UK

Adilia Warris

UK

Gregory Waryasz

USA

Edward Waters

Australia

Laura Waters

UK

Chand Wattal

India

Rachel Wattier

USA

Andy Wearn

New Zealand

Adam Weber

USA

Heinrich Weber

Australia
Scott Weese

Canada

Junni Wei

China

Louis Weiss

USA

Scott Weiss

USA

Christian Wejse

Denmark

Christina Welinder-Olsson

Sweden

Xi Wen

USA

Nicolas Wentzensen

USA

Lars Westblade

USA

Dawn Wetzel

USA

Jaclyn White

USA

Nathan White

USA

Andrew Christopher Whitelaw South Africa

Timothy Wiemken

USA

Annelies Wilder-Smith

Singapore

Katalin Wilkinson

South Africa

Birgit Willinger

Austria

Matthias Willmann

Germany

Peter Wilson

UK

John Wingard

USA

Brita Askeland Winje

Norway

Andrea Winkler

Australia 


\begin{tabular}{|c|c|c|}
\hline $\begin{array}{l}\text { Kevin Winthrop } \\
\text { USA }\end{array}$ & $\begin{array}{l}\text { Biao Xu } \\
\text { China }\end{array}$ & $\begin{array}{l}\text { Barnaby Young } \\
\text { Singapore }\end{array}$ \\
\hline $\begin{array}{l}\text { Carl Heinz Wirsing Von Koenig } \\
\text { Germany }\end{array}$ & $\begin{array}{l}\text { Howard Xu } \\
\text { USA }\end{array}$ & $\begin{array}{l}\text { Jo-Anne Young } \\
\text { USA }\end{array}$ \\
\hline $\begin{array}{l}\text { Desalegn Woldeyohannes } \\
\text { Ethiopia }\end{array}$ & $\begin{array}{l}\text { Kari Yacisin } \\
\text { USA }\end{array}$ & $\begin{array}{l}\text { Kung-Chia Young } \\
\text { Taiwan }\end{array}$ \\
\hline $\begin{array}{l}\text { Hilary Wolf } \\
\text { USA }\end{array}$ & $\begin{array}{l}\text { Laith Yakob } \\
\text { UK }\end{array}$ & $\begin{array}{l}\text { Zobair Younossi } \\
\text { USA }\end{array}$ \\
\hline $\begin{array}{l}\text { Elke Wollants } \\
\text { Belgium }\end{array}$ & $\begin{array}{l}\text { Jie Yan } \\
\text { China }\end{array}$ & $\begin{array}{l}\text { Chong-Jen Yu } \\
\text { Taiwan }\end{array}$ \\
\hline $\begin{array}{l}\text { Nicole Wolter } \\
\text { South Africa }\end{array}$ & $\begin{array}{l}\text { Yongping Yan } \\
\text { China }\end{array}$ & $\begin{array}{l}\text { Luo-Ting Yu } \\
\text { China }\end{array}$ \\
\hline $\begin{array}{l}\text { Karen Wong } \\
\text { USA }\end{array}$ & $\begin{array}{l}\text { Frances Yang } \\
\text { USA }\end{array}$ & $\begin{array}{l}\text { Chee Fu Yung } \\
\text { Singapore }\end{array}$ \\
\hline $\begin{array}{l}\text { Ngai Sze Wong } \\
\text { Hong Kong }\end{array}$ & $\begin{array}{l}\text { Samuel Yang } \\
\text { USA }\end{array}$ & $\begin{array}{l}\text { Hans L Zaaijer } \\
\text { The Netherlands }\end{array}$ \\
\hline $\begin{array}{l}\text { Christopher Wood } \\
\text { USA }\end{array}$ & $\begin{array}{l}\text { Stephanie Yanow } \\
\text { Canada }\end{array}$ & $\begin{array}{l}\text { Mauro Zaccarelli } \\
\text { Italy }\end{array}$ \\
\hline $\begin{array}{l}\text { Darcy Wooten } \\
\text { USA }\end{array}$ & $\begin{array}{l}\text { Cedric Yansouni } \\
\text { Canada }\end{array}$ & $\begin{array}{l}\text { Keivan Zandi } \\
\text { Malaysia }\end{array}$ \\
\hline $\begin{array}{l}\text { Dan Wootton } \\
\text { UK }\end{array}$ & $\begin{array}{l}\text { Satomi Yara } \\
\text { Japan }\end{array}$ & $\begin{array}{l}\text { Isabella Zanella } \\
\text { Italy }\end{array}$ \\
\hline $\begin{array}{l}\text { Michael Worobey } \\
\text { USA }\end{array}$ & $\begin{array}{l}\text { Masaki Yasukawa } \\
\text { Japan }\end{array}$ & $\begin{array}{l}\text { Maurizio Zazzi } \\
\text { Italy }\end{array}$ \\
\hline $\begin{array}{l}\text { David Wright } \\
\text { UK }\end{array}$ & $\begin{array}{l}\text { Michio Yasunami } \\
\text { Japan }\end{array}$ & $\begin{array}{l}\text { Jonathan Zelner } \\
\text { USA }\end{array}$ \\
\hline $\begin{array}{l}\text { M Wroblewska } \\
\text { Poland }\end{array}$ & $\begin{array}{l}\text { Tom Yates } \\
\text { UK }\end{array}$ & $\begin{array}{l}\text { Erliang Zeng } \\
\text { USA }\end{array}$ \\
\hline $\begin{array}{l}\text { Bin Wu } \\
\text { China }\end{array}$ & $\begin{array}{l}\text { Barbara Yawn } \\
\text { USA }\end{array}$ & $\begin{array}{l}\text { Nicola Zetola } \\
\text { Botswana }\end{array}$ \\
\hline $\begin{array}{l}\text { David Bin-Chia Wu } \\
\text { Taiwan }\end{array}$ & $\begin{array}{l}\text { Kojo Yeboah-Antwi } \\
\text { USA }\end{array}$ & $\begin{array}{l}\text { Chiyu Zhang } \\
\text { China }\end{array}$ \\
\hline $\begin{array}{l}\text { Lawrence Shih Hsin Wu } \\
\text { Taiwan }\end{array}$ & $\begin{array}{l}\text { Venkat Laxmi Yeruva } \\
\text { USA }\end{array}$ & $\begin{array}{l}\text { Di Zhang } \\
\text { USA }\end{array}$ \\
\hline $\begin{array}{l}\text { Ming-Shiang Wu } \\
\text { Taiwan }\end{array}$ & $\begin{array}{l}\text { Ahmet Yilmaz Çoban } \\
\text { Turkey }\end{array}$ & $\begin{array}{l}\text { Jun Zhang } \\
\text { China }\end{array}$ \\
\hline $\begin{array}{l}\text { Peng Wu } \\
\text { Hong Kong }\end{array}$ & $\begin{array}{l}\text { Jae-Joon Yim } \\
\text { South Korea }\end{array}$ & $\begin{array}{l}\text { Yanan Zhao } \\
\text { USA }\end{array}$ \\
\hline $\begin{array}{l}\text { Lihua Xiao } \\
\text { USA }\end{array}$ & $\begin{array}{l}\text { Cyril Yip } \\
\text { Hong Kong }\end{array}$ & $\begin{array}{l}\text { Pingyu Zhou } \\
\text { China }\end{array}$ \\
\hline $\begin{array}{l}\text { Xianhong Xie } \\
\text { USA }\end{array}$ & $\begin{array}{l}\text { Jean Cyr Yombi } \\
\text { Belgium }\end{array}$ & $\begin{array}{l}\text { Yanhe Zhou } \\
\text { China }\end{array}$ \\
\hline $\begin{array}{l}\text { Zheng Xing } \\
\text { USA }\end{array}$ & $\begin{array}{l}\text { Lay-Myint Yoshida } \\
\text { Japan }\end{array}$ & $\begin{array}{l}\text { Xing-Quan Zhu } \\
\text { China }\end{array}$ \\
\hline
\end{tabular}


Yefei Zhu

China

Panayiotis Ziakas

USA

David Zielinski

Canada

Hisham Ziglam

UK
Marya Zilberberg

USA

Daniel Zinder USA

Walter Zingg

Switzerland

York Zoellner

Germany
Huachun Zou

Australia

Benjamin Zuber

France

Simbarashe Peter Zvada

South Africa

Alice Zwerling

USA 